\title{
Dissolution: A Quality Control Test vs. A Bioequivalence Test
}

Vinod P. Shah, Ph. D.

Senior Research Scientist, Office of Pharmaceutical Science, Center for Drug Evaluation and Research, Food and Drug Administration, Rockville, MD

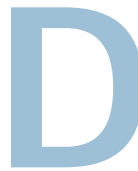

issolution testing over the last quarter century has emerged as a highly valuable in vitro test to characterize drug product performance. It is an important tool in drug development and in quality control (QC). In drug development it is used to guide formulation development and to select an appropriate formulation for in vivo testing. In quality control dissolution testing is used as a batch-to-batch quality test before the product is released into the market place. For oral solid dosage forms, the dissolution test forms the basisfor setting specifications (test, methodology, acceptance criteria) to allow batch release. It is used to identify bioavailability (BA) problems and to assess the need for further bioequivalence (BE) studies relative to scale-up and post-approval changes (SUPAC), where it can function as a signal of bioinequivalence. At present, almost all solid oral dosage forms require dissolution as a QC test before a product is introduced into the market place. For the test to be useful, it should be simple, reliable and reproducible and should be able to discriminate between different degrees of product performance. The value of the test issignificantly enhanced when product performance is evaluated as a function of time,i.e., when the dissolution profile is determined rather than a single point determination. Because of the importance of dissolution, FDA has developed dissolution related guidances that provide information and recommendations on the development of dissolution test methodology,setting dissolution specifications and the regulatory applications of dissolution testing $(1,2)$.

Over the last decade, with the increasing knowledge in dissolution scientific principles and mechanisms, the dissolution test has turned a new leaf. The dissolution test is used as a surrogate marker for bioequivalence test, as evidenced from a recent Biopharmaceutics Classification System (BCS) guidance (3). The BCS guidance takes into account three major factors, dissolution,solubility and intestinal permeability, that govern the rate and extent of drug absorption from immediate release solid dosage forms. The BCS provides a scientific framework for classifying drug substances based on aqueous solubility and intestinal permeability, and email: shahvi@cder.fda.gov

in combination with dissolution data, providesa rationale for biowaiver of immediate release (IR) drug products. In addition, the General Bioavailability and Bioequivalence guidance (4) allows biowaivers for lower strength(s) of immediate release as well as modified release drug products based on formulation proportionality and dissolution profile comparison.

These changes in bioequivalence requirements, moving away from in vivo biostudy requirements in certain cases and relying more on dissolution test, clearly establish a change in dissolution test applications. In all cases where dissolution test is used as a BEtest, an anchor with a bioavailable product is established. With the evolution and advances in dissolution technology and the understanding of scientific principles and mechanism of dissolution test results, a clear trend has emerged where dissolution test has moved from traditional quality control test to a surrogate in vitro bioequivalence test.This represents a shift in dissolution thought process and a new regulatory perspective on dissolution. The dissolution test has moved away from traditional QC test to a surrogate in vitro BE test (Table 1).In several instances, a biowaiver is indicated based on dissolution profile comparison.

\section{Table 1.}

\section{Changing Role of Dissolution Science}

New Regulatory Perspectives in Dissolution

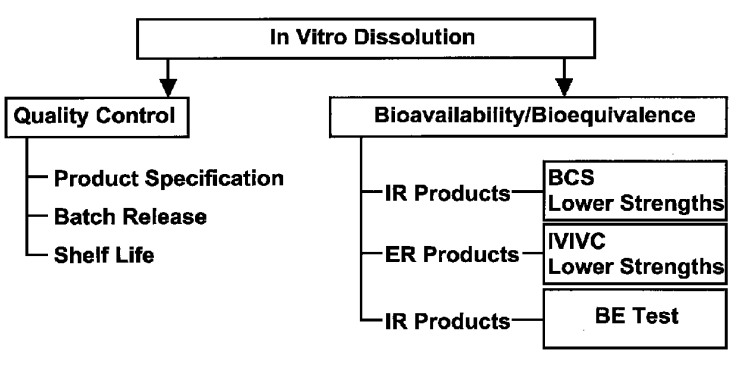

FDA has adopted a simple mathematical approach for dissolution profile comparison, termed similarity factor, $f_{2}(1,6,7)$. Regulatory interest is to know how similar the two dissolution profile curves are, and for this reason, the $f_{2}$ comparison has been the focus in Agency guidances. When the two profiles are identical, $f_{2}=100$. An average 
difference of $10 \%$ at all measured time points results in a $\mathrm{f}_{2}$ value of 50.FDA has set a public stand ard for the $f_{2}$ value of between 50-100 to indicate similarity between two dissolution profiles, thus implying similar in vivo product performance.

When dissolution is used as a QC test for IR products, it is generally a single point dissolution test and is represented as $\mathrm{X} \%$ dissolved in Y minutes. However, when the dissolution test is used as a BEtest, a dissolution profile is generated for a profile comparison with a bioavailable product.

The value of dissolution test can be further enhanced in developing countries where it can be used as a "bioequivalence test." A question to consid er is: "Can dissolution test alone be used as a BE test for approval of IR products in developing countries?" Generally in developing countries, the technology and other resources are very limited to conduct an appropriate in vivo bioequivalence study.Under these circumstances, appropriate dissolution studies, such as, profile comparison between the local generic product and the reference product in $\mathrm{pH} 1.2,4.5$ and 6.8 media under mild test conditions, basket method at $100 \mathrm{rpm}$ or paddle method at $50 \mathrm{rpm}$, may be used to assure product quality. This appears to be a practical approach that can be easily considered and adopted for BE test in developing countries.

In conclusion, in vitro dissolution testing is increasingly relied upon to assure product performance and product quality.The in vitro test procedure is a simple and economical method that can be utilized effectively in developing countries to assure acceptable drug product quality.Dissolution testing is currently used as a QC test, (generally single point for immediate release products and 3-to-4 points for extended release products), as well as an in vitro bioequivalence (BE) test (generally dissolution profile and profile comparison).Significance of both the tests are different, and should be carefully separated.

\section{References:}

1. Guidance for Industry: Dissolution Testing of Immediate Release Solid Oral Dosage Form, August 1997.

2. Guidance for Industry: Extended Release Solid Oral Dosage Forms:Development, Evaluation and Application of In Vitro/In Vivo Correlations, September 1997.

3. Guidance for Industry: Waiver of In Vivo Bioavailability and Bioequivalence Studies for Immediate-Release
Solid Oral Dosage Forms Based on a Biopharmaceutics Classification System, August 2000.

4. Guidance for Industry: Bioavailability and Bioequivalence Studies for Orally Administered Drug Products - General Considerations, October 2000.

5. G.L.Amidon,H.Lennernas, V. P. Shah and J. R. Crison. A theoretical basis for a biopharmaceutics drug classification:The correlation of in vitro drug product dissolution and in vivo bioavailability. Pharm. Research.12: 413-420 (1995)

6. V.P.Shah, Y.Tsong, P. Sathe and J .P. Liu. In vitro dissolution profile comparison - statistics and analysis of the similarity factor, f2. Pharm. Research.15:889-896,1998.

7.V.P.Shah, Y.Tsong, P. Sathe and R. L. Williams. Dissolution profile comparison using similarity factor, $\mathrm{f} 2$. Dissolution Technologies. 6(3),15,1999. 